\title{
Microfluidic Rheometer Based on Hydrodynamic Focusing
}

\author{
Nam-Trung Nguyen§, Yit-Fatt Yap, Agung Sumargo \\ $\dagger$ School of Mechanical and Aerospace Engineering, Nanyang Technological \\ University, 50 Nanyang Avenue, Singapore 639798
}

\begin{abstract}
This paper reports the concept and the optimization of a microfluidic rheometer based on hydrodynamic focusing. In our microfluidic rheometer, a sample stream is sandwiched between two sheath streams. The width of the middle stream depends on the viscosity ratio and the flow rate ratio of the liquids involved. Fixing the flow rate ratio and using a known Newtonian liquid for the sheath streams, the viscosity and the shear stress of the sample stream can be determined by measuring its width and using a prediction algorithm that uses the known channel geometries, fluid properties, flow rates and the focused width as input parameters. The optimization reveals that measurement channel with high aspect ratio is more suitable for sample liquid with viscosity higher than the reference value. For sample liquid with viscosity of the same order of magnitude or lower than the reference, a low aspect ratio is more suitable. Furthermore, the measurement range and the relative error can be improved by adjusting the flow rate ratio between the core stream and the sheath stream. A microfluidic device was fabricated in polymethylmethacrylate (PMMA). Using this device, viscosities of deionized (DI) water and polyethylene oxide (PEO) solutions were measured and compared with results obtained from a commercial rheometer.
\end{abstract}

$\S$ To whom correspondence should be addressed (mntnguyen@ntu.edu.sg) 


\section{Introduction}

Viscosity is one of the key properties of a liquid. Fast, on-line measurement of the viscosity is important for many industrial processes, especially for combinatorial chemistry and food processing industry. Viscosity as function of shear rate was measured with a number of rheometry concepts. Commercially available rheometers can generally categorized as shear rheometer and extensional rheometer. In a shear rheometer, the shear rate is measured directly or indirectly with different configurations such as parallel plates, cone plates, rotational cylinder of capillary tubes. In an extensional rheometer, the viscosity and elongation rate are determined when the liquid is pulled apart. Extensional rheometers can be used for highly viscous fluids such as polymer melts [1].

Capillary rheometers belong to the shear rheometer family. The liquid sample is forced through a capillary with known geometry. The two main parameters of this system are the flow rate and the pressure. If one parameter is fixed, the other one can be measured. With the known dimensions, the viscosity and the shear rate can be estimated from the known or measured flow rate and pressure drop. Varying the applied pressure or flow rate allows the measurement of viscosity over a wide range of shear stress. Microfluidic technologies allow the implementation of capillary rheometry on a single chip. Galambos and Forster [2] used hydrodynamic spreading in a T junction device to measure the viscosity of an unknown Newtonian fluid using a known miscible reference fluid. Fluorescent dye was mixed with the reference fluid for the detection with a fluorescence microscope. The interface position between the two fluids can be measured near the channel inlet and at relatively high flow rates because diffusive mixing is negligible. The pressure drop was measured indirectly based on the interface position. Guillot et al. applied the same concept to two immiscible fluids and extended it to the measurement of non-Newtonian fluids [3]. This concept needs a microscope and image processing software to evaluate the results. Srivastava and Burns [4] used passive capillary filling to measure the viscosity of the liquid. The flow rate was measured by monitoring the meniscus position, while the pressure was estimated based on the interfacial tension and the contact angle at the meniscus. Kang et al. [5] used external pressure sensors to measure the pressure drop across microchannels. Zimmerman et al. [6] showed that one microfluidic experiment can be used to evaluate the constitutive relationship for power-law non-Newtonian fluids. This concept was demonstrated later by Bandulasena et al. [7] using micro particle image velocimetry.

Recently, we have investigated the hydrodynamic focusing effect of Newtonian liquids and derived a simple relation between the focused width and the viscosity [8] as well as the flow rate ratio [9]. We also have demonstrated in-situ measurement of the focused width using a micro optofluidic device [10]. The previous results indicate the possibility of designing a microfluidic rheometer based on hydrodynamic focusing. Furthermore, we also have demonstrated microfluidic sensors for the measurement of surface tension [11] and interfacial tension [12]. The possibility of making a single 
microfluidic device for the measurement of different fluid properties such as viscosity, surface tension and interfacial tension motivates this current work. The present paper reports the numerical method for predicting the viscosity and the shear stress from the measured width of the focused stream. We also reports the experimental results of this concept for non-Newtonian fluids. The focused width was measured with a fluorescent microscope and a CCD camera.

\section{Measurement concept}

\subsection{Model and governing equations}

Figure 1 shows the cross section of a rectangular channel with the width $a$ and the height $b$. Three different fluids 1, 2 and 3, flow in the channel in a stratified manner. These parallel fluid layers are separated by interfaces denoted as $\Gamma_{12}$ and $\Gamma_{23}$. For simplicity, these interfaces are presently assumed to be flat planes. The widths of fluids 1,2 and 3 are $\delta_{1}, \delta_{2}$ and $\delta_{3}$, respectively. The restriction of plane interfaces can be liberated if required to allow a more realistic curved interfaces for immiscible liquids. The flow becomes unidirectional in the fully-developed region. Diffusive mixing effects are ignored, thus the focused middle stream is considered as immiscible to the sheath streams.

A one-fluid formulation is used for the numerical procedure. The domain of interest is assumed to consist of one special fluid. The properties of this special fluid vary spatially. The property of this special fluid at a given spatial location is equal to that of the fluid (1, 2 or 3$)$ occupying that particular location. Thus, the distribution of fluids 1, 2 and 3 in the domain needs to be identified. Each fluid is identified by a level-set function [13], a signed distance function with positive values within the region occupied by that particular fluid but negative elsewhere. A total of three distance functions are required:

$$
\begin{gathered}
\zeta_{1}(\mathbf{x}) \begin{cases}<0 & \text { if } \mathbf{x} \in \Omega_{2} \cup \Omega_{3} \\
=0 & \text { if } \mathrm{x} \in \Gamma_{12} \\
>0 & \text { if } \mathrm{x} \in \Omega_{1}\end{cases} \\
\zeta_{2}(\mathbf{x}) \begin{cases}<0 & \text { if } \mathrm{x} \in \Omega_{2} \cup \Omega_{3} \\
=0 & \text { if } \mathrm{x} \in \Gamma_{12} \cup \Gamma_{23} \\
>0 & \text { if } \mathrm{x} \in \Omega_{2}\end{cases} \\
\zeta_{3}(\mathbf{x}) \begin{cases}<0 & \text { if } \mathrm{x} \in \Omega_{1} \cup \Omega_{2} \\
=0 & \text { if } \mathrm{x} \in \Gamma_{23} \\
>0 & \text { if } \mathrm{x} \in \Omega_{3}\end{cases}
\end{gathered}
$$

where $\mathbf{x}$ is the location vector. Three smeared Heaviside functions are defined based on these level-set functions to facilitate the calculation of the properties of the special 


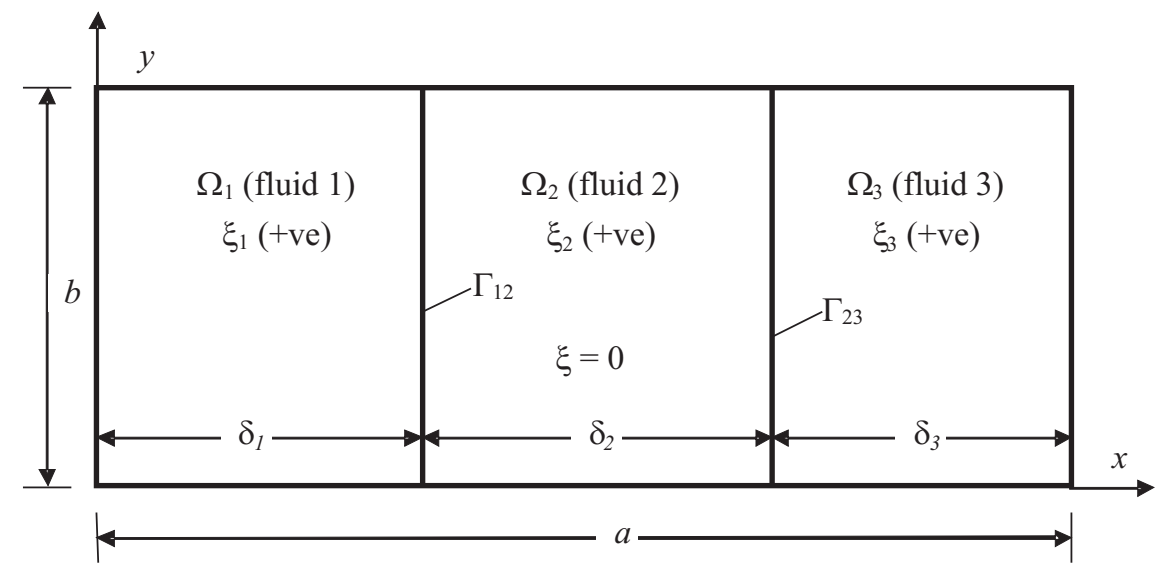

(a)

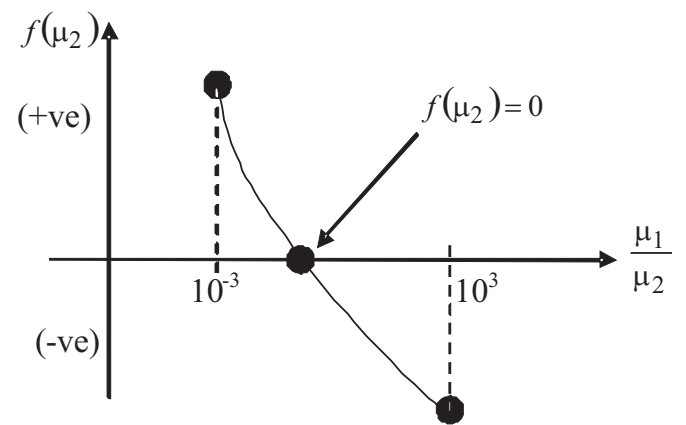

(b)

Figure 1. The model of hydrodynamic focusing with three stratified fluid streams: (a) computational domains, (b) two initial guesses to initialize bisection method.

fluid.

$$
H_{n}\left(\zeta_{n}\right)= \begin{cases}0 & \text { if } \zeta_{n}<\varepsilon \\ \frac{\zeta_{n}+\varepsilon}{2 \varepsilon}+\frac{1}{2 \pi} \sin \left(\frac{\pi \zeta_{n}}{\varepsilon}\right) & \text { if }\left|\zeta_{n}\right| \leq \varepsilon \\ 1 & \text { if } \zeta_{n}>\varepsilon\end{cases}
$$

where $n=1,2,3$. The smeared Heaviside function allows a smooth change of the special fluid properties across an interface of thickness $2 \varepsilon$ where $\varepsilon$ is a fraction of the control volume size in the discretized Navier-Stokes equations. The viscosity at any given location $\mathbf{x}$ can now be conveniently evaluated with:

$$
\frac{1}{\mu(\mathbf{x})}=\frac{H_{1}}{\mu_{1}}+\frac{H_{2}}{\mu_{2}}+\frac{H_{3}}{\mu_{3}}
$$

Subsequently, the volumetric flow rates of fluids 1, 2 and 3 can be computed as:

$$
\dot{Q}_{n}=\int_{A} w H_{n} \mathrm{~d} A
$$

where $n=1,2,3, A$ is the cross section of the channel and $w$ is the velocity component in the $z$-direction.

The Navier-Stokes equation for a unidirectional incompressible flow in the $z$ direction $\mathbf{u}=[u, v, w]$ with $u=v=0$ reduces to

$$
-\frac{\mathrm{d} p}{\mathrm{~d} z}+\nabla \cdot(\mu \nabla w)=0
$$


with the boundary condition $w=0 \forall \mathbf{x} \in \partial\left(\Omega_{1} \cup \Omega_{2} \cup \Omega_{3}\right)$. This equation represents a 2-D unidirectional, axial steady laminar simplification of the Cauchy momentum equation, and can also be solved with series expansion [14].

It is worth noted that for given viscosity ratios $\mu_{1} / \mu_{2}$ and $\mu_{3} / \mu_{2}$, a pressure gradient $\mathrm{d} p / \mathrm{d} z$ only sets the exact flow rates $\dot{Q}_{1}$ and $\dot{Q}_{2}$ in (6). It does not alter the ratio $\dot{Q}_{1} / \dot{Q}_{2}$ and $\dot{Q}_{3} / \dot{Q}_{2}$. Therefore, the pressure gradient is set to $\mathrm{d} p / \mathrm{d} z=-1$ for the sake of computational convenience. The problem for the microfluidic rheometer is formulated as "for given $\mu_{1}, \mu_{3}, K_{12}$ and $K_{13}$, find $\mu_{2}$ that the flow field satisfies $\dot{Q}_{1} / \dot{Q}_{2}=K_{12}$ and $\dot{Q}_{3} / \dot{Q}_{2}=K_{32}$ ". These constraints are equivalent to $f_{A}=0$ and $f_{B}=0$ where $f_{A}=\dot{Q}_{1} / \dot{Q}_{2}-K_{12}$ and $f_{B}=\dot{Q}_{3} / \dot{Q}_{2}-K_{32}$. The functions $f_{A}$ and $f_{B}$ behave similarly as $\dot{Q}_{2}$ varies due a variation in $\mu_{2}$. A flow rate $\dot{Q}_{2}$ smaller than the correct value results in both positive $f_{A}$ and $f_{B}$. The reverse occurs, as a flow rate $\dot{Q}_{2}$ larger than the correct value results in both negative $f_{A}$ and $f_{B}$. Given the similar behaviour of these functions, we can combine them into a single equation as:

$$
f\left(\mu_{2}\right)=\frac{\dot{Q}_{1}}{\dot{Q}_{2}}-K_{12}+\frac{\dot{Q}_{3}}{\dot{Q}_{2}}-K_{32}
$$

With this new function, the problem for the numerical algorithm reduces to "find $\mu_{2}$ such that $f\left(\mu_{2}\right)=0 "$.

\subsection{Prediction algorithm for the microfluidic rheometer}

Based on the above analysis, the following algorithm was derived for predicting the viscosity of the unknown fluid from other known parameters:

(i) For an experimentally determined interface $\Gamma_{12}$ and $\Gamma_{23}$, compute $\zeta_{n}(\mathbf{x})$ for $n=$ $1,2,3$.

(ii) Calculate $H_{n}(\mathbf{x})$ for $n=1,2,3$ using equation (4).

(iii) Guess $\mu_{2}$, calculate $\mu(\mathbf{x})$ using equation (5).

(iv) Solve the Navier-Stokes equation (7) for $w(\mathbf{x})$.

(v) Compute $\dot{Q}_{1}, \dot{Q}_{2}$ and $\dot{Q}_{3}$ using equation (6).

(vi) Compute $f\left(\mu_{2}\right)$ from equation (8).

(vii) Check if $\left|f\left(\mu_{2}\right)\right|$ is less then the allowable error $\Delta f$, if not then improve the guess of $\mu_{2}$ and repeat steps (i) to (vii).

The simplified Navier-Stokes equation is solved using a finite volume method with a second order spatial discretization. Step (vii) is accomplished with a bisection method. The two initial guesses of $\mu_{2}$ to initial the method are $\mu_{2}=10^{4} \mu_{1}$ and $\mu_{2}=10^{-4} \mu_{1}$ as shown in Figure 1(b). The allowable error for the function $f\left(\mu_{2}\right)$ is set to $\Delta f=10^{-7}$. These initial guess are arbitrarily defined, other criteria can also be employed. For instance, the convergence criteria can be the rate of change in $\mu_{2}$ in two consecutive iterations:

$$
\left|\frac{\mu_{2, \text { new }}-\mu_{2, \text { old }}}{\mu_{2, \text { new }}}\right|<\Delta f
$$


With the recovered velocity distribution and viscosity, the average shear rate of the focused stream is estimated as:

$$
\dot{\gamma}=\frac{1}{A_{2}} \iint_{\Omega_{2}}|\nabla w| \mathrm{d} A
$$

where $|\nabla u|=\sqrt{(\partial w / \partial x)^{2}+(\partial w / \partial y)^{2}}$ and $A_{2}=\iint_{A} H_{2} \mathrm{~d} A$. The standard deviation of the local shear rate in the focused stream is given by

$$
\Delta \dot{\gamma}=\frac{1}{A_{2}} \iint_{\Omega_{2}} \sqrt{|\nabla w|^{2}-\dot{\gamma}^{2}} \mathrm{~d} A
$$

Since the local shear rate in the focused stream ranges from 0 to a maximum value at the channel wall, the standard deviation should be on the same order of magnitude as the averaged shear rate.

\subsection{Validation of the prediction algorithm}

The following two validation cases were calculated: three streams with the same viscosity and three streams with different viscosities in a rectangular microchannel. The analytical solution for the velocity distribution of a single stream in a rectangular channel cross section $a \times b$ (Figure 1) is [15]:

$$
w=-\frac{1}{2 \mu} \frac{\mathrm{d} p}{\mathrm{~d} z} b^{2}\left[1-\left(\frac{y}{b}\right)^{2}+4 \sum_{k=1}^{\infty} \frac{(-1)^{k}}{\alpha_{k}^{3}} \frac{\cosh \left(\alpha_{k} x / b\right)}{\cosh \left(\alpha_{k} a / b\right)} \cos \left(\alpha_{k} y / b\right)\right]
$$

where $\alpha_{k}=(2 k-1) \pi / 2$. For $a=1, b=1, \mu_{1}=\mu_{2}=\mu_{3}=1$ and the corresponding widths $\delta_{1}=0.2, \delta_{2}=0.3$ and $\delta_{3}=0.5$, the flow rate of each stream can be determined from the analytical solution (9) as $\dot{Q}_{1}=0.112 \dot{Q}, \dot{Q}_{2}=0.388 \dot{Q}$ and $\dot{Q}_{3}=0.500 \dot{Q}$ where the total flow rate is $\dot{Q}=\dot{Q}_{1}+\dot{Q}_{2}+\dot{Q}_{3}$. Thus, the prediction algorithm described in the previous section was applied to the given parameters $a=b=1, \mu_{1}=\mu_{3}=1$, $\delta_{1}=0.2, \delta_{2}=0.3$, and $\delta_{3}=0.5$. Figure 2 shows the recovered velocity distribution and the predicted viscosity as function of mesh size. At a mesh number of $300 \times 300$, the viscosity of the middle stream $\mu_{2}=1$ was well predicted.

In the case of different viscosities $\mu_{1}=1, \mu_{2}=2$ and $\mu_{3}=3$, the velocity distribution with $\delta_{1}=0.2, \delta_{2}=0.3$ and $\delta_{3}=0.5$, the Navier-Stokes equation can only be solved numerically. The resulting flow rates are $\dot{Q}_{1}=0.165 \dot{Q}, \dot{Q}_{2}=0.424 \dot{Q}$ and $\dot{Q}_{3}=0.411 \dot{Q}$, where $\dot{Q}$ is the total flow rate. For the prediction algorithm, all parameters and the flow rates are given, the viscosity $\mu_{2}$ needs to be recovered. Figure 2 shows the recovered velocity distribution and the predicted viscosity $\mu_{2}$ as a function of the mesh numbers in each dimension. The results also show that the viscosity of the middle stream $\mu_{2}=2$ is well predicted with a $300 \times 300$ mesh.

\subsection{Error analysis}

In their previous work on using hydrodynamic spreading to measure the viscosity, Guillot et al. [3] used the ratio between the standard deviation of the shear rate (11) and the average shear rate (10) to evaluate the accuracy of the predicted shear rate. This analysis 


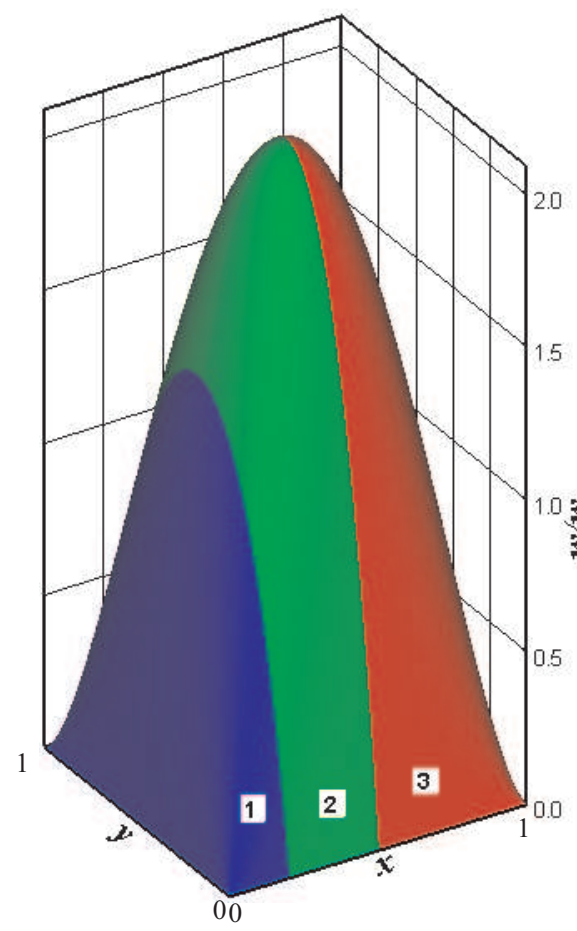

(a)

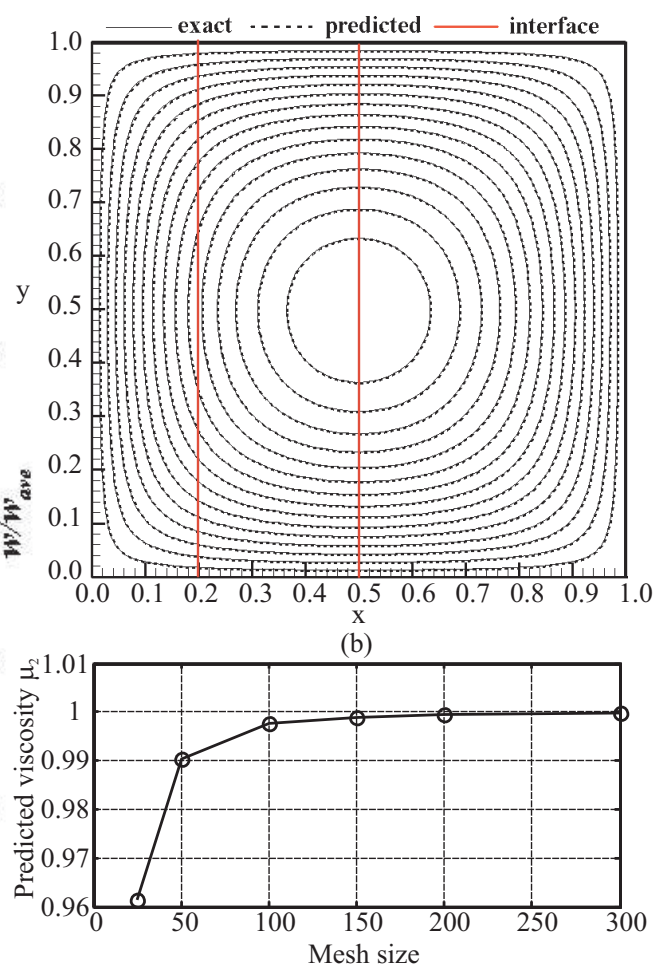

(c)

Figure 2. Velocity distribution in a channel with $a=b=1, \mu_{1}=\mu_{2}=\mu_{3}=1$, $\delta_{1}=0.2, \delta_{2}=0.3$, and $\delta_{3}=0.5$ :(a) recovered velocity distribution; (b) comparison between the predicted solution and the analytical solution; (c) predicted viscosity $\mu_{2}$ as a function of mesh numbers on each dimension.

used a fixed microchannel geometry and the flow rate ratio as well as the viscosity ratio as the optimization parameters. However in a practical situation, the uncertainties in the measured focused width directly affect the accuracy of the measured viscosity. In this error analysis, we consider the focused width as the main source of measurement error. The aim of the optimization is to minimize this error for different viscosity ranges. Two optimization parameters are investigated: the aspect ratio of the measurement channel $b: a$ and the flow rate ratio between the focused stream and one side stream $\dot{Q}_{2} / \dot{Q}_{1}$. Using the above numerical model, the normalized predicted viscosity $\mu_{2}^{*}=\mu_{2} / \mu_{1}$ was determined as a function of the normalized focused width $\delta_{2}^{*}=\delta_{2} / a$, Figure $4(\mathrm{a})$. The first order derivative of this function [Figure 4(b)] represents the sensitivity of the predicted viscosity against the measured focused width. The relative error of the measured viscosity $\Delta \mu_{2}$ can be estimated from the error of the width measurement $\Delta \delta_{2}$ as

$$
\frac{\Delta \mu_{2}}{\mu_{2}}=\frac{\mathrm{d} \mu_{2}^{*}}{\mathrm{~d} \delta_{2}^{*}} \frac{\Delta \delta_{2}}{a} \frac{\mu_{1}}{\mu_{2}}=\frac{\mathrm{d} \mu_{2}^{*}}{\mathrm{~d} \delta_{2}^{*}} \frac{\Delta \delta_{2}}{a} \frac{1}{\mu_{2}^{*}}
$$

The results of the first order derivative $\mathrm{d} \mu_{2}^{*} / \mathrm{d} \delta_{2}^{*}$ clearly show that the predicted viscosity is more sensitive to the change in focused width at a higher aspect ratio. According to (13), the relative error of the measured viscosity can be reduced if $\mathrm{d} \mu_{2}^{*} / \mathrm{d} \delta_{2}^{*}$ and $\mu_{2}^{*}$ are kept small and large, respectively. Figure 4 shows that a low aspect ratio 


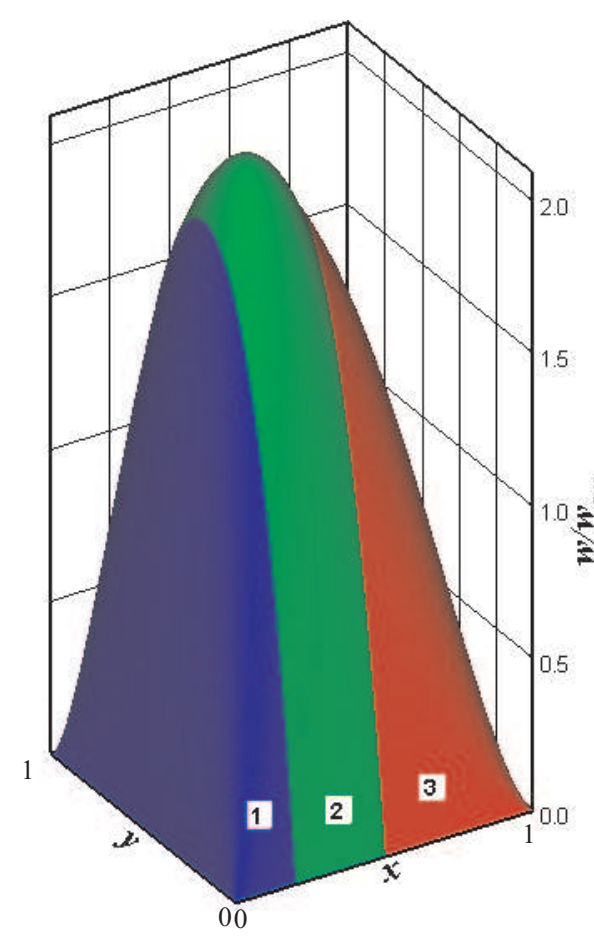

(a)

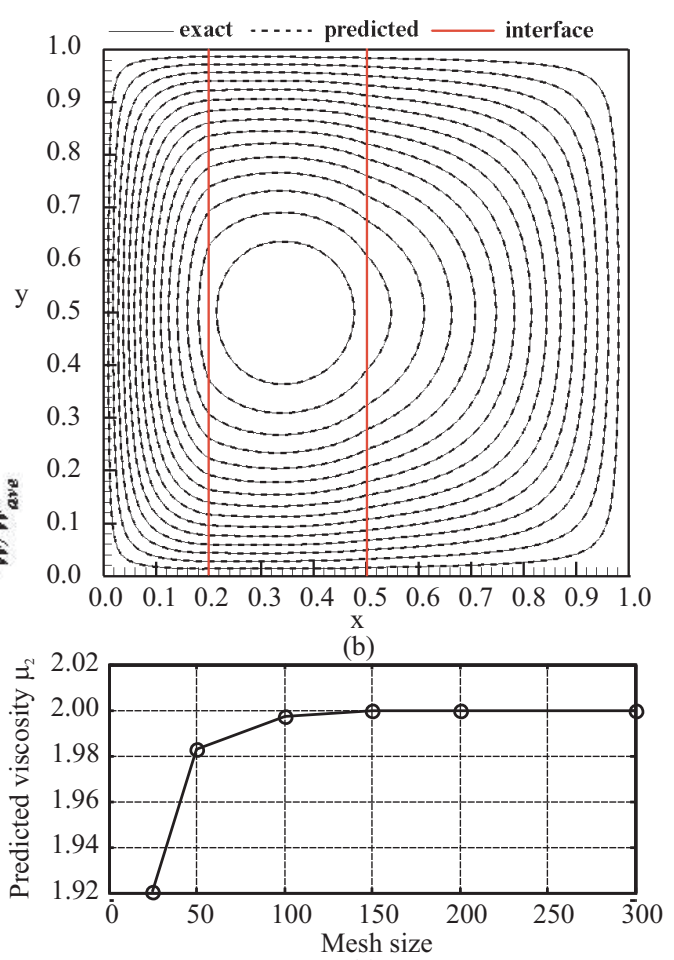

(c)

Figure 3. Velocity distribution in a channel with $a=b=1, \mu_{1}=1, \mu_{2}=2, \mu_{3}=3$, $\delta_{1}=0.2, \delta_{2}=0.3$, and $\delta_{3}=0.5$ :(a) recovered velocity distribution; (b)comparison between the predicted solution and the exact numerical solution; (c) predicted viscosity $\mu_{2}$ as a function of mesh numbers on each dimension.

$b / a$ is more suitable for sample liquids with viscosity on the same order of magnitude as the reference liquid. These liquids will have a unreasonable high error at higher aspect ratio due to the large $\mathrm{d} \mu_{2}^{*} / \mathrm{d} \delta_{2}^{*}$. According to (13), if the viscosity of the sample liquid is 2 or 3 orders of magnitude higher than the reference liquid, a high aspect ratio $b / a$ is more suitable because it can cover this viscosity range and can keep the error low due to the large $\mu^{*}$. More details on the error analysis for our experiments in a measurement channel with the aspect ratio $b / a \approx 1.6$ are discussed in the next section.

Figure 5 shows relationships between the measured viscosity $\mu_{2}^{*}$ and the focused width $\delta_{2}^{*}$ at different flow rate ratios $\dot{Q}_{2} / \dot{Q}_{1}$. The results show that a sample fluid with viscosity higher than the reference should have a low flow rate ratio $\dot{Q}_{2} / \dot{Q}_{1}$ to have the right viscosity range and a small error. Conversely, a sample fluid with viscosity lower than the reference should have a high flow rate ratio $\dot{Q}_{2} / \dot{Q}_{1}$.

\section{Experimental setup and results}

The microfluidic rheometer was fabricated in polymethylmethacrylate (PMMA) using laser micromachining. The device consists of three 1-mm-thick sheets. The middle layer contains the measurement channel with three inlets. The top layer has the access holes for the inlets and outlets. The bottom layer seals the whole channel network. The 


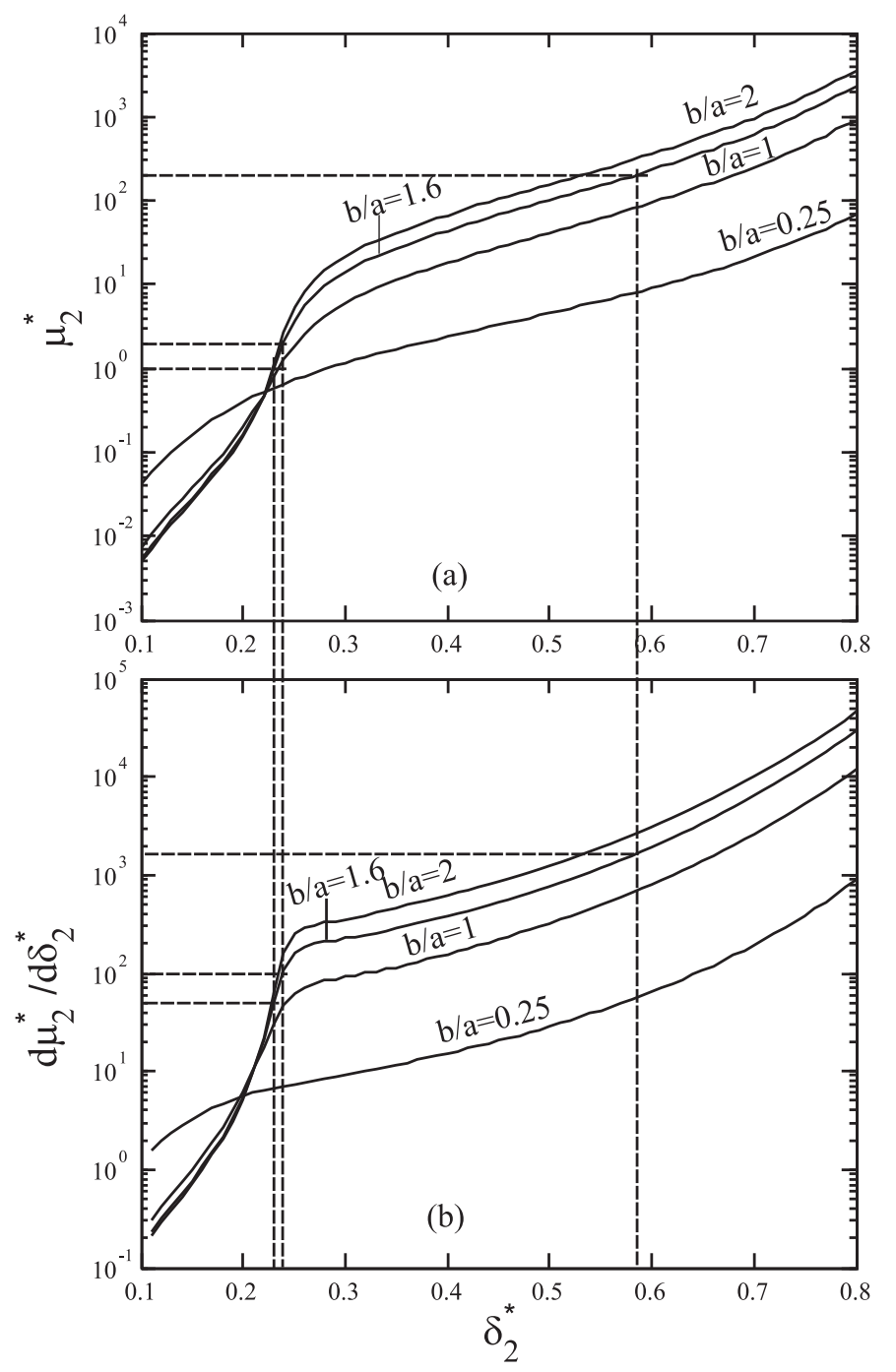

Figure 4. Sensitivity analysis $\left(\mu_{1}=\mu_{3}, \dot{Q}_{1}=\dot{Q}_{2}=\dot{Q}_{3}\right)$ : (a) normalized viscosity $\mu_{2}^{*}$ as function of normalized width $\delta_{2}^{*}$; (b) first order derivative of normalized viscosity $\mathrm{d} \mu_{2}^{*} / \mathrm{d} \delta_{2}^{*}$ as function of normalized width $\delta_{2}^{*}$.

channel structures and the access holes were first designed using CorelDraw (Corel Co., Canada). A commercial $\mathrm{CO}_{2}$ laser system (Universal M-300 Laser Platform, Universal Laser Systems Inc., Arizona, USA) were used to cut through the 1-mm PMMA sheets. The height and the width of the fabricated microchannels are $a=1 \mathrm{~mm}$ and $b=625 \mu \mathrm{m}$.

In our experiments, de-ionized (DI) water with a fluorescent dye was used as the reference fluid. The fluorescent dye is fluorescein disodium salt $\mathrm{C}_{2} \mathrm{OH}_{10} \mathrm{Na}_{2} \mathrm{O}_{5}$ (also called Acid Yellow 73 or C.I. 45350). The dye has an excitation wave length and an emission wave length of $490 \mathrm{~nm}$ and $520 \mathrm{~nm}$, respectively. The measured area was illuminated with a mercury lamp with an epi-fluorescent attachment of type Nikon B-2A was used (excitation filter for 450-490 nm, dichroic mirror for $505 \mathrm{~nm}$ and an emission filter for $520 \mathrm{~nm}$ ). DI water and solutions with $0.1 \mathrm{w} \%$ and $2 \mathrm{w} \%$ polyethyleneoxide (PEO) in DI-water was used as sample fluids. All experiments were carried out in an air 

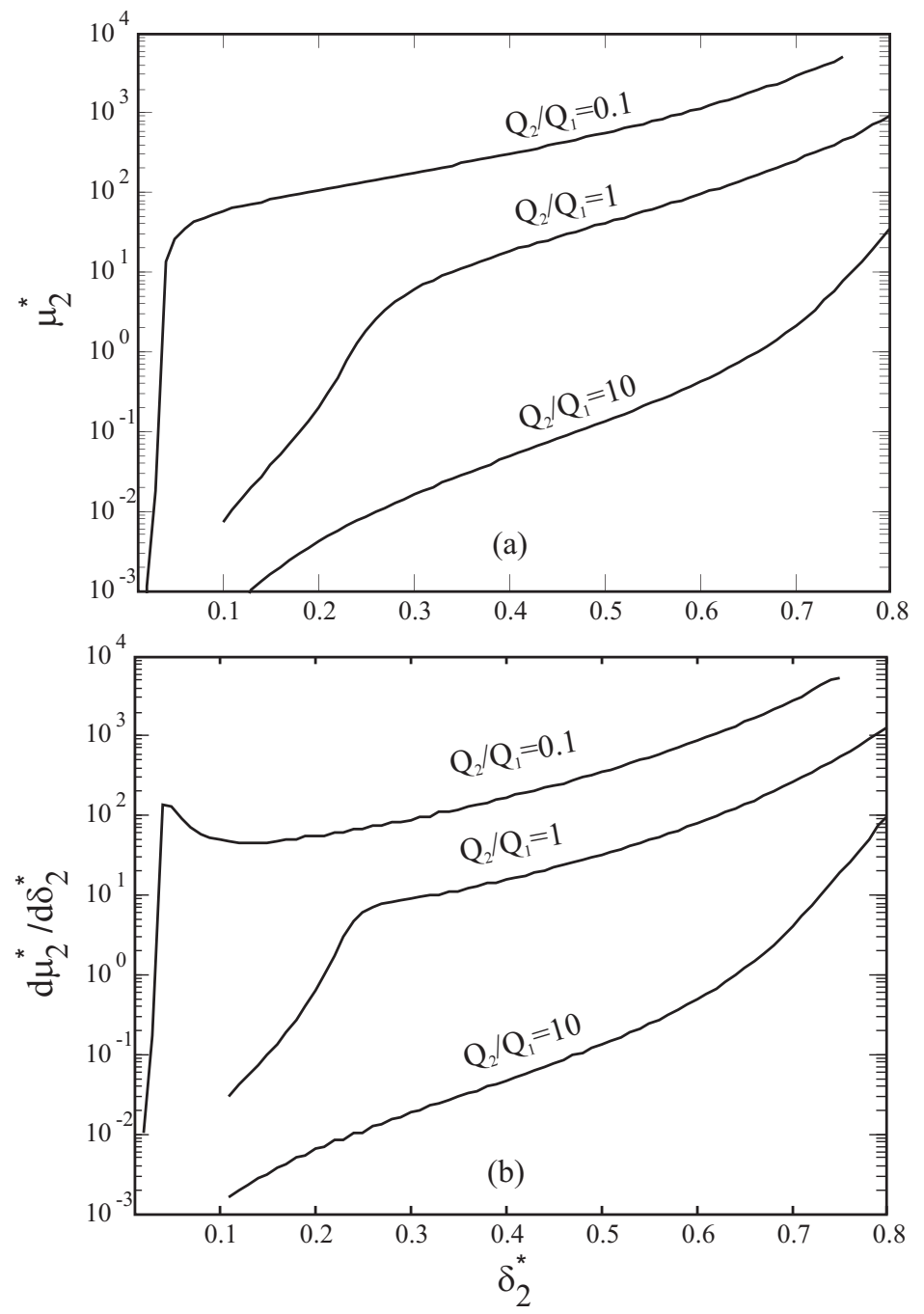

Figure 5. Sensitivity analysis $\left(\mu_{1}=\mu_{3}, \dot{Q}_{1}=\dot{Q}_{3}, b / a=1\right)$ : (a) normalized predicted viscosity $\mu_{2}^{*}$ as function of normalized measured width $\delta_{2}^{*}$; (b) first order derivative of normalized predicted viscosity $\mathrm{d} \mu_{2}^{*} / \mathrm{d} \delta_{2}^{*}$ as function of normalized measured width $\delta_{2}^{*}$.

conditioned room at $25{ }^{\circ} \mathrm{C}$. The viscosity versus shear rate data of the reference fluid and the sample fluids were collected with a commercial plate-plate rheometer (Advanced Rheometry Expasion System, Rheometric Scientific Inc., NJ, USA). The reference fluid showed a Newtonian behavior with a viscosity of $0.922 \times 10^{-3}$ Pa.sec. Three identical syringes (Hamilton $500 \mu \mathrm{l}$ gas tight) were filled with the reference fluids as well as the sample and placed on a syringe pump (KS 230, KD Scientific). The syringe pump drive three syringes (Hamilton, $500 \mu \mathrm{l}$ ) at the same time. Flow rates ranging from $1 \mathrm{ml} / \mathrm{h}$ to $150 \mathrm{ml} / \mathrm{h}$ were used in the experiments. Silicone tubing were used to connect the syringes with the inlets of the microfluidic device. A sensitive CCD camera (HiSense MKII) was used to capture the fluorescent image of the measurement channel. Figure 6 shows the experimental setup for measuring the width of the sample stream. With this setup, both sheath streams and the sample stream can be driven with the same flow rates. The shear rates are adjusted by varying the flow rate of the three streams. To 


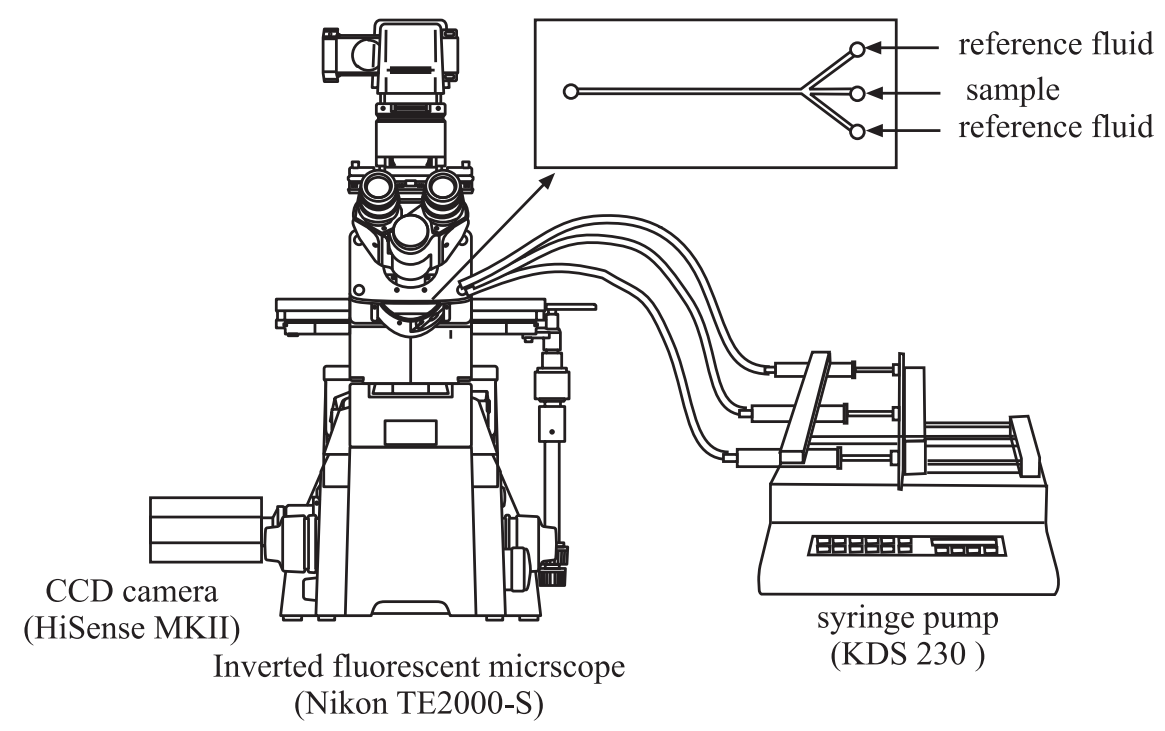

Figure 6. Experimental setup.

Table 1. Error estimation for the viscosity measurement.

\begin{tabular}{llll}
\hline Sample & $\mu_{2}^{*}$ & $\mathrm{~d} \mu_{2}^{*} / d \delta_{2}^{*}$ & Relative error $\Delta \mu_{2} / \mu_{2}$ \\
\hline DI Water & $\approx 1$ & $\approx 50$ & $24 \%$ \\
PE0 0.1 wt\% & $\approx 2$ & $\approx 100$ & $24 \%$ \\
PE0 2 wt\% & $\approx 200$ & $\approx 2000$ & $4.8 \%$ \\
\hline
\end{tabular}

make sure that molecular diffusion is minimum and the interface is stable, the images were taken near the entrance of the measurement channel.

The images captured by the CCD camera was transferred to a PC. The width of the sample stream was evaluated using a customized program written in MATLAB. Figure 7(a) shows the typical fluorescent images of the measurement channel. While the sheath streams with fluorescent dye appear white, the sample stream is black. This configuration allows the simple measurement of the sample stream. A line was sampled across the middle stream. The typical intensity distribution along such as line is shown in Figure 7(a). The gradient of the intensity values was subsequently calculated. The distance between the two maxima of the gradient profile is taken as the width of the focused stream, Figure 7(a). The standard deviation of the width measurement is about $\Delta \delta_{2}=3 \mu \mathrm{m}$. The viscosities of the DI water, $0.1 \mathrm{wt} \%$ PEO solution and $2 \mathrm{wt} \%$ $\mathrm{PEO}$ solution at high shear rates are approximately $1 \times 10^{-3} \mathrm{~Pa} . \mathrm{sec}, 2 \times 10^{-3} \mathrm{~Pa}$.sec and $2 \times 10^{-1}$ Pa.sec, respectively. Using the numerical results depicted in Fig. 4 and equation (13), the relative error for DI water, $0.1 \mathrm{wt} \%$ PEO solution and $2 \mathrm{wt} \%$ PEO solution are $10 \%, 1.25 \%$ and $2.5 \%$. The details of the error calculation are listed in table 1. It's apparent that the relative error for liquids that are more viscous then the reference liquid is smaller.

To estimate the flow condition of the sample flow as well as the influence of 


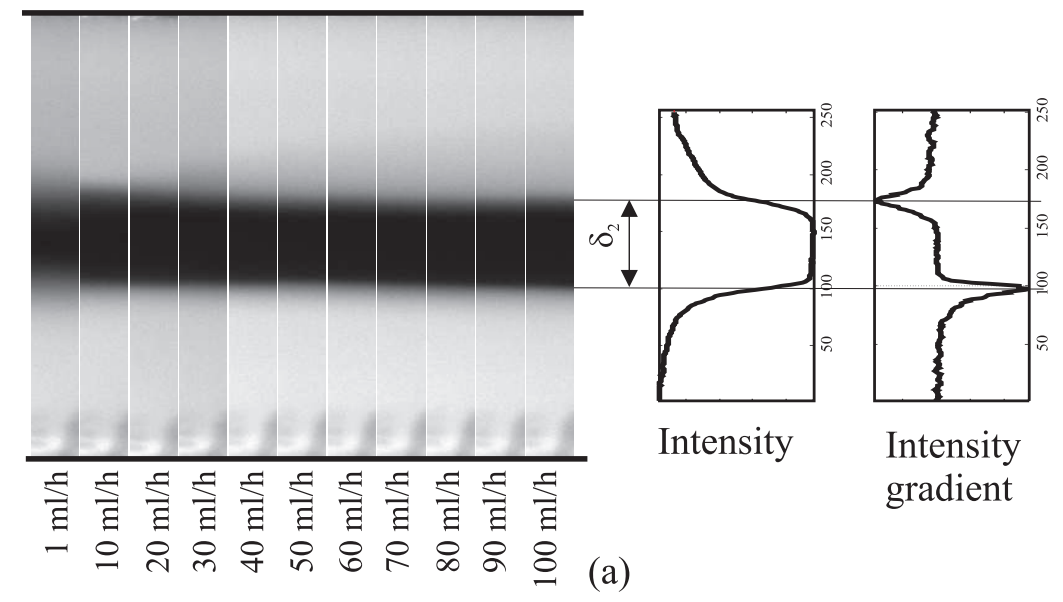

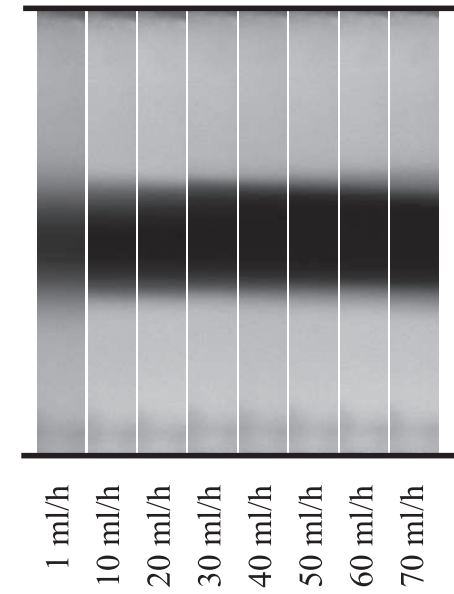

(b)

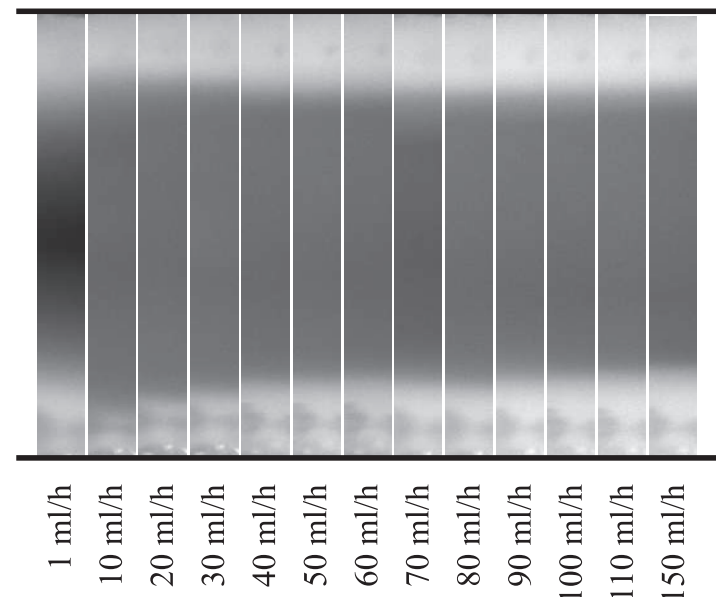

(c)

Figure 7. Measurement of the width of the focused stream with an unknown viscosity: (a) Fluorescent images for water as sample fluid, the typical intensity distribution and the intensity gradient; (b) Fluorescent images of PEO $0.1 \mathrm{wt} \%$ as sample fluid; (c) Fluorescent images of PEO $2 \mathrm{wt} \%$ as sample fluid.

molecular diffusion on the measurement, the Reynolds number $\operatorname{Re}=\rho_{2} w_{2} D_{\mathrm{h}} / \mu_{2}$ and the Peclet number $\mathrm{Pe}=w_{2} D_{\mathrm{h}} / D_{2}$ were estimated and listed in Table 2 . The hydraulic diameter of the sample stream $\left(D_{\mathrm{h}}=4 A / U\right)$, where $A$ is the cross-sectional areas and $U$ is the perimeter, was calculated based on its width and channel height. The width of DI-water, PEO $0.1 \mathrm{wt} \%$ and PEO $2 \mathrm{wt} \%$ were taken at high flow rates and are approximately $145 \mu \mathrm{m}, 150 \mu \mathrm{m}$ and $380 \mu \mathrm{m}$, respectively. The calculation uses the viscosity values according to Table 1 with $\mu_{1}=10^{-3}$ Pa.sec and a density of $1000 \mathrm{~kg} / \mathrm{m}^{3}$. The diffusion coefficients of the fluorescent dye in DI-water, PEO $0.1 \mathrm{wt} \%$ and PEO 2 wt $\%$ are $1.8 \times 10^{-9} \mathrm{~m}^{2} / \mathrm{sec}[8], 0.9 \times 10^{-9} \mathrm{~m}^{2} / \mathrm{sec}$ and $0.9 \times 10^{-11} \mathrm{~m}^{2} / \mathrm{sec}$, respectively. The values for the PEO solutions are estimated based on the Einstein's theory that the diffusion coefficient is inversely proportional to the dynamic viscosity. Table 2 shows that the flows in the experiments are laminar and the Peclet number are high enough to neglect molecular diffusion. 
Table 2. The range of Reynolds number and Peclet number in the experiments.

\begin{tabular}{lllllll}
\hline $\begin{array}{l}\dot{Q} \\
(\mathrm{ml} / \mathrm{h})\end{array}$ & $\begin{array}{l}\text { Re } \\
(\text { DI water })\end{array}$ & $\begin{array}{l}\text { Pe } \\
(\text { DI water })\end{array}$ & $\begin{array}{l}\text { Re } \\
(\text { PEO 0.1 wt\% })\end{array}$ & $\begin{array}{l}\text { Pe } \\
(\text { PEO 0.1 wt\% })\end{array}$ & $\begin{array}{l}\text { Re } \\
(\text { PEO 2 wt\% })\end{array}$ & $\begin{array}{l}\text { Pe } \\
(\text { PEO 2 wt\% })\end{array}$ \\
\hline 1 & 0.485 & $2.70 \times 10^{2}$ & 0.241 & $5.37 \times 10^{2}$ & 0.201 & $4.47 \times 10^{4}$ \\
10 & 4.85 & $2.70 \times 10^{3}$ & 2.41 & $5.37 \times 10^{3}$ & 2.01 & $4.47 \times 10^{5}$ \\
1 & 48.5 & $2.70 \times 10^{4}$ & 24.1 & $5.37 \times 10^{4}$ & 20.1 & $4.47 \times 10^{6}$ \\
\hline
\end{tabular}

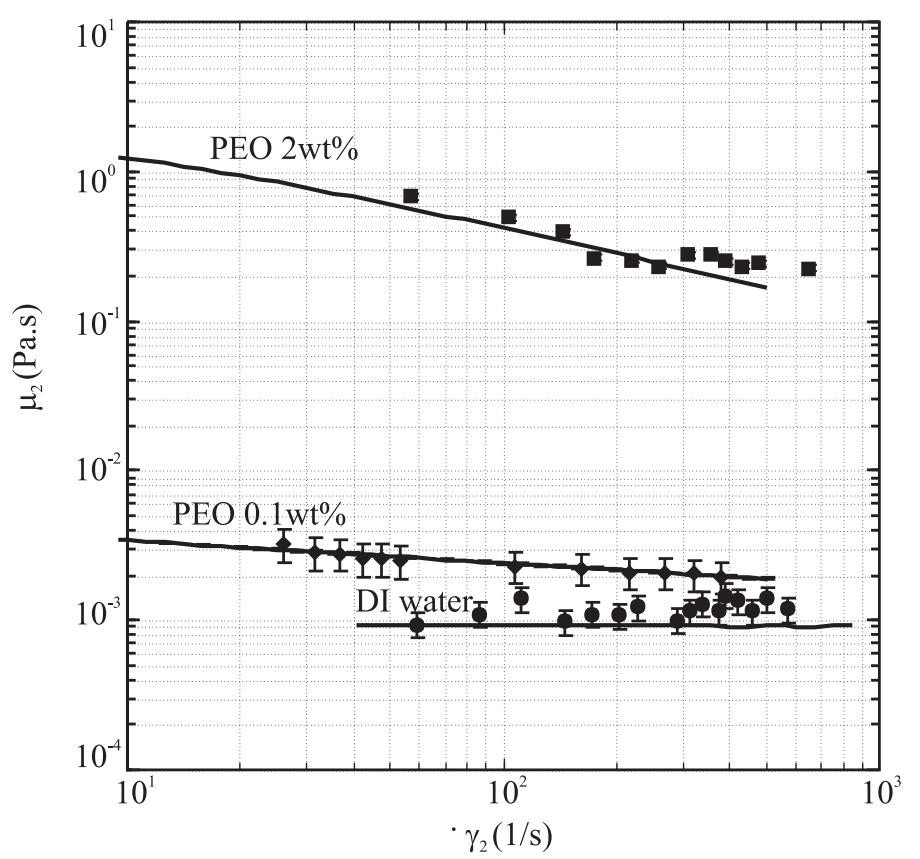

Figure 8. Viscosities predicted from the measured focused width: (a) water, (b) PEO $0.1 \mathrm{wt} \%$, (c) PEO 2 wt\%.

The measured widths and other parameters such as channel geometry, flow rates of each stream and viscosity of the reference fluids are passed to the prediction program using an input file in ASCII format. The results are also saved in an output file in ASCII format. Figure 8 shows the results obtained with the microfluidic rheometer compared to that obtained with a commercial plate-plate rheometer. For both PEO solutions, the width of the focused stream decreases with an increasing flow rate. This behavior reflects the decreasing viscosity at an increasing shear rate.

There are some uncertainties in our current method. The advantage of using miscible liquids is the wide range of flow rates that can be employed. The draw back is the diffusion of the fluorescent dye which lead to a diffuse region where the interface can only estimated based on the gradient of the distribution of the dye concentration. Furthermore, the prediction algorithm is based on a Newtonian model, where the focused stream is modeled with a shear-independent viscosity. That means, the algorithm not only averages the shear rate but also the viscosity over the cross section of the focused stream. A further problem is that the viscoelastic property of a non-Newtonian fluid 
causes instabilities at high shear rate. In the past, we have used this property to design micromixers $[16,17]$. The instability may destroy the stable interfaces between the streams.

\section{Conclusions}

This paper reports a prediction algorithm and a prototype for a microfluidic rheometer based on hydrodynamic focusing. The measurement concept is based on a three-stream system. The sheath streams consist of a reference Newtonian fluid with a known viscosity. Fluorescent dye was diluted in this reference fluid for the later measurement with a fluorescent microscope. The sample stream with the unknown viscosity and the the two sheath streams were introduced into the microfluidic device using a syringe pump. The prediction algorithm recovers the viscosity of the sample fluid from the known channel geometry, flow rates and the width of the focused stream. The shear rate was predicted from the recovered velocity profile and the known interface position. The optimization reveals that measurement channel with high aspect ratio is more suitable for sample liquids with viscosity much higher than the reference value. For sample liquid with viscosity of the same order of magnitude or lower than the reference, a low aspect ratio is recommended. The measurement range and the relative error can be improved buy adjusting the flow rate ratio between the core stream and the sheath stream. A sample liquid with higher viscosity would need a lower flow rate ratio, while a higher flow rate ratio is more suitable for sample liquids with low viscosity. The results show the possibility of designing a microfluidic rheometer with hydrodynamic focusing instead of hydrodynamic spreading as reported previously by other groups. The optofluidic concept [10] would make the detection concept on chip possible without a fluorescent microscope and a CCD camera. With a known channel geometry, flow rates and reference liquids, the prediction algorithm is easily embedded in a micro controller or a signal processor. If the device is to be used only as a viscometer for Newtonian fluids, the prediction algorithm can be replaced by the much faster polynomial fitting of the functions depicted in Figures 4(a) and 5(a). Thus, a portable hand-held rheometer can be designed using the concept reported here.

\section{Acknowledgement}

NT Nguyen would like to thank the Ministry of Education, Singapore for its financial support under project RG22/05"Characterisation of Low Viscosity Elastic Fluids in Microscale".

\section{References}

[1] Walters K 1975 Rheometry (Chapman \& Hall) ISBN 0412120909

[2] Galambos P and Forster F 1998 Optical micro-fluidic viscometer Intl Mech Eng Cong Exp. Anaheim, CA., ASME, Dynamic Systems and Control Division (Publication) DSC 66, 187-191. 
[3] Guillot P, Panizza P, Salmon J-B, Joanicot M, Colin A, Bruneau C-H, Colin T 2006 Viscosimeter on a microfluidic chip Langmuir 22 6438-6445

[4] Srivastava N and Burns MA 2006 Analysis of non-Newtonian liquids using a microfluidic capillary viscometer J. Anal Chem $\mathbf{7 8}$ 1690-1696

[5] Kang K, Lee LJ, Koelling KW 2005 High shear microfluidics and its application in rheological measurement Exp Fluids 38 222-232

[6] Zimmerman WB, Rees JM, Craven TJ 2006 Rheometry of non-Newtonian electrokinetic flow in a microchannel T-junction Microfluid. Nanofluid. 2 481-492

[7] Bandalusena HCH, Zimmerman WB, Rees JM 2008 An inverse methology for the rheology of a power law non-Newtonian fluid J. Mech. Eng. Sci. in press

[8] Wu Z, Nguyen NT 2005 Hydrodynamic focusing in microchannels under consideration of diffusive dispersion: theories and experiments Sensors and Actuators B: Chemical 107 965-974

[9] Nguyen NT 2005 Micromixers - Fundmentals, Design and Fabrication, William Andrew, 150-153

[10] Nguyen NT, Kong TF, Goh JH, Low CLN 2007 Micro optofluidic splitter and switch based on hydrodynamic spreading Journal of Micromechanics and Microengineering 17 2169-2174

[11] Nguyen NT, Lassemono S, Chollet FA, Yang C 2006 Microfluidic sensor for dynamic surface tension measurement IEE Proceedings-Nanobiotechnology 153 102-106

[12] Nguyen NT, Lassemono S, Chollet FA, Yang C 2006 Interfacial tension measurement with an optofluidic sensor IEEE Sensors Journal 7 692-697

[13] Sethian JA 1999 Level Set Methods and Fast Marching Methods: Evolving Interfaces in Computational Geometry, Fluid Mechanics, Computer Vision, and Materials Science 2nd ed. (Cambridge University Press, Cambridge)

[14] Zimmerman WB 2004 On the resistance of a spherical particle settling in a tube of viscous fluid International Journal of Engineering Science 42 1753-1778

[15] Shah RK and London AL 1978 Laminar flow forced convection in ducts (Academic Press, New York)

[16] Gan HY, Lam YC, Nguyen NT 2006 A polymer-based device for efficient mixing of viscoelastic fluids Applied Physics Letter $\mathbf{8 8} 224103$

[17] Gan HY, Lam YC, Nguyen NT, Yang C, Tam KC 2007 Efficient mixing of visco-elastic fluids in a microchannel at low Reynolds number Microfluidics and Nanofluidics 3 101-108 